\title{
Extrinsic Allergic Alveolitis
}

National Cancer Institute

\section{Source}

National Cancer Institute. Extrinsic Allergic Alveolitis. NCI Thesaurus. Code C34369.

An inflammatory interstitial lung disease caused by hypersensitivity reaction to inhalation or ing estion of antigens. The antigens are usually related to the patient's occupation. It can present as an acute illness with flu-like symptoms, subacute with repeated episodes of pneumonia, or chronic with dyspnea and productive cough. The majority of patients recover following the cessation of the exposure to the antigen that causes the disease. Chronic exposure may eventually progress to interstitial lung fibrosis. 